\title{
The Giant Magellan Telescope phasing system: Algorithms and performance simulations
}

\author{
Fernando Quirós-Pacheco*a, Antonin H. Bouchez ${ }^{\mathrm{a}}$, Rodolphe Conan ${ }^{\mathrm{a}}$, \\ Brian A. McLeod ${ }^{\mathrm{b}}$, David Schwartz \\ ${ }^{a}$ GMTO Organization, 465 North Halstead St, Suite 250, Pasadena, CA 91107, USA \\ ${ }^{\mathrm{b}}$ Smithsonian Astrophysical Observatory, 60 Garden St, MS 20, Cambridge, MA 02138, USA
}

\begin{abstract}
The $25.4 \mathrm{~m}$ Giant Magellan Telescope (GMT) consists of seven $8.4 \mathrm{~m}$ primary mirror (M1) segments with matching segmentation of the Gregorian secondary mirror (M2). When operating the telescope in the diffraction-limited Adaptive Optics (AO) observing modes, the M1-M2 pairs of segments must be phased to a small fraction of the observing wavelength. The first part of this paper presents a revised Phasing / Active Optics control strategy that ensures the phased condition across the scientific field of view, combining measurements of different wavefront sensing channels available in the Acquisition Guiding and Wavefront Sensing (AGWS) system. The second part of this paper presents a preliminary evaluation of the effects of wind buffeting on the phasing performance of the telescope in the LTAO mode. In this observing mode, edge sensors on M1 and M2 sense segment piston disturbances and drive their fast compensation with the Adaptive Secondary Mirror (ASM).
\end{abstract}

Keywords: Giant Magellan Telescope, Extremely Large Telescopes, co-phasing, adaptive optics, wave-front control

\section{INTRODUCTION}

The GMT [1] is a doubly segmented telescope, with a primary mirror (M1) composed of seven circular 8.4 m segments, and two interchangeable secondary mirrors (M2) - a Fast Steering Mirror (FSM) and an Adaptive Secondary Mirror (ASM) - composed of seven circular segments matched 1:1 to the segments of M1.

Since both M1 and M2 are segmented, the total optical path of each M1-M2 segment pair must be equalized to a small fraction of the observing wavelength $(0.9-25 \mu \mathrm{m})$ to meet image quality requirements in the two diffraction-limited AO observing modes, namely the Natural Guide-star AO (NGAO) and the Laser Tomography AO (LTAO) modes. The Natural Seeing (NS) mode does not require active phasing control.

The phasing system relies on continuous measurements of phasing errors performed at slow rates $(\sim 0.03 \mathrm{~Hz})$ by dedicated phasing sensors in the Acquisition Guiding and Wavefront Sensing (AGWS) system (see $\S 1.1$ ). In Section 2 of this paper, we present a revised Phasing/Active Optics control strategy that combines measurements of different wavefront sensing channels available in the AGWS to ensure the phased condition across the scientific field of view ( $<90$ " in diameter).

When operating in the NGAO mode, the AGWS is responsible for the initial phasing of the seven segment pairs. Phasing control is then taken over by the Natural Guide star Wavefront sensor (NGWS) [2], featuring a pyramid wavefront sensor, which provides a fast measurement rate $(\sim 1 \mathrm{kHz})$ necessary to control the residual on-axis phasing errors induced by the atmospheric turbulence, telescope vibrations, and wind buffeting. During the course of an observation, the AGWS stays on-line to monitor phasing errors in the science field, but also to provide a phasing correction in the event a segment phase piston (SPP) error "escapes" the limited capture range of the NGWS.

When operating in the LTAO mode, the AGWS keeps the telescope phased over the course of an observation at slow rates. There is no optical sensor in the Laser Tomography Wavefront Sensor (LTWS) [3] that can provide fast correction of turbulence-induced SPP errors in this observing mode. While turbulence-induced SPP errors remain uncorrected, the phasing system relies on M1 edge sensors (M1ES) and M2 edge sensors (M2ES) to measure phasing errors induced by wind buffeting and vibrations at medium rates $(\sim 500 \mathrm{~Hz})$. In Section 3 of this paper, we present preliminary simulation results of the wind buffeting rejection capabilities that edge sensors can enable.

*fquiros@gmto.org 


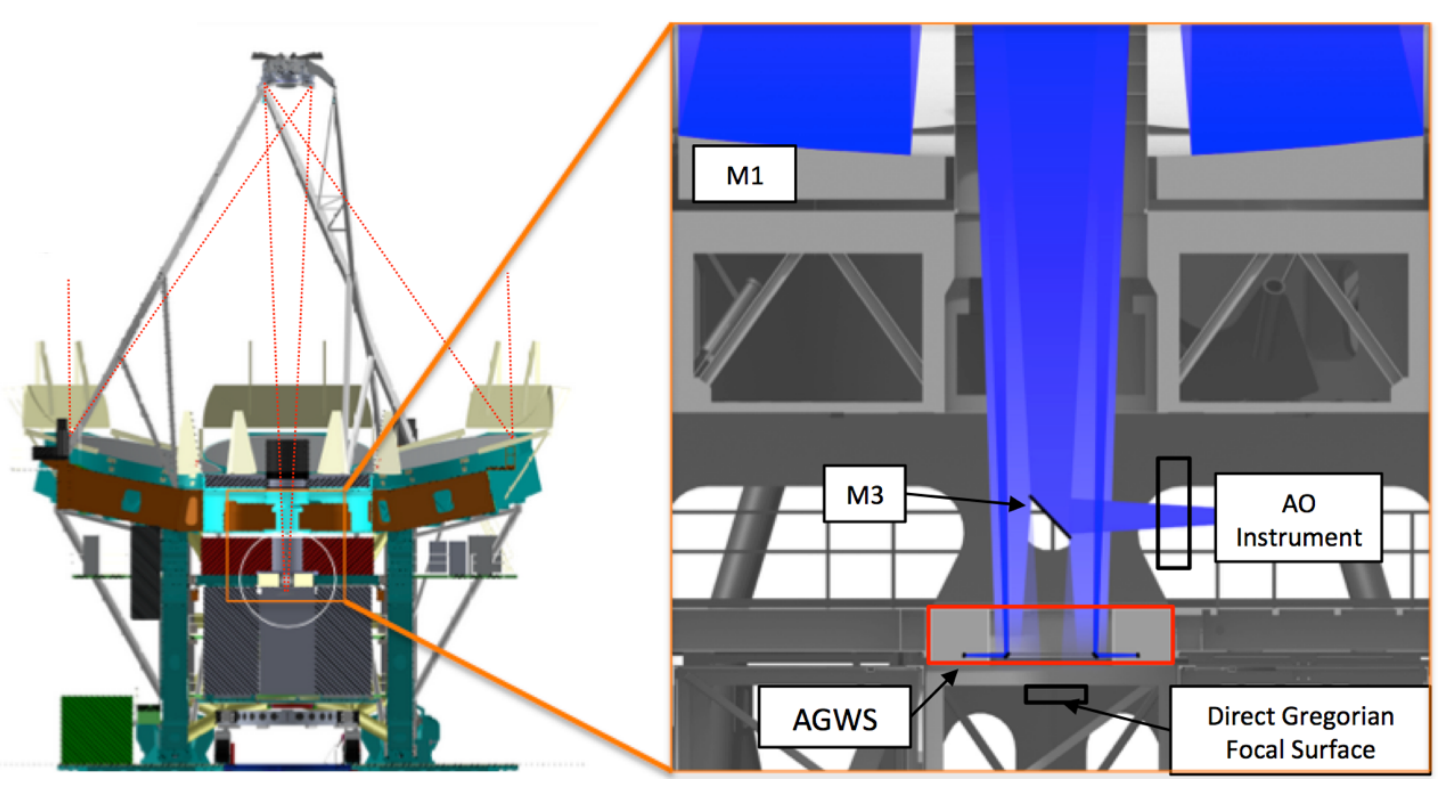

Figure 1. GMT in the Folded Port (FP) mode. The AGWS is located below the tertiary mirror (M3) at the top of the Gregorian Instrument Rotator (GIR), $\sim 0.5 \mathrm{~m}$ above the Direct Gregorian focal surface. At this location, the AGWS star probes can patrol an annular field of view ranging from 5 to 10 arcminutes off-axis.

\subsection{Acquisition Guiding and Wavefront Sensor (AGWS)}

The Acquisition Guiding and Wavefront Sensing System (AGWS), designed by the Smithsonian Astrophysical Observatory, is responsible for making the measurements needed by the GMT active optics and phasing systems to keep the seven-segment pairs co-aligned, phased, pointing in the correct direction, and conforming to the correct mirror shape.

The AGWS features four identical star probes that can be positioned anywhere in the 10' radius field, but only between 5' and 10' radius field when the tertiary (M3) is deployed to feed the Folded Port (FP) instruments (Figure 1). These are the instruments that will benefit from NGAO/LTAO correction.

Each AGWS star probe will contain five selectable optical channels (see Figure 2): four visible (operating from 600$900 \mathrm{~nm}$ ) and one IR (operating in J band). The four visible channels are: a $30 \operatorname{arcsec~FoV~acquisition~camera;~a~} 48 \times 48$ Shack-Hartmann wavefront sensor capable both of long time-scale active optics measurements and ground-layer wavefront sensing at $100 \mathrm{~Hz}$; a fast $(\sim 1 \mathrm{kHz})$ guide channel for natural guide star tip/tilt measurement during LTAO; and a 7 subaperture channel for segment tip/tilt sensing. The IR channel is the phasing sensor that will be fed by a dichroic beamsplitter. The phasing sensor is a Dispersed Fringe Sensor (DFS), and it is described in detail in van Dam et al. [4].
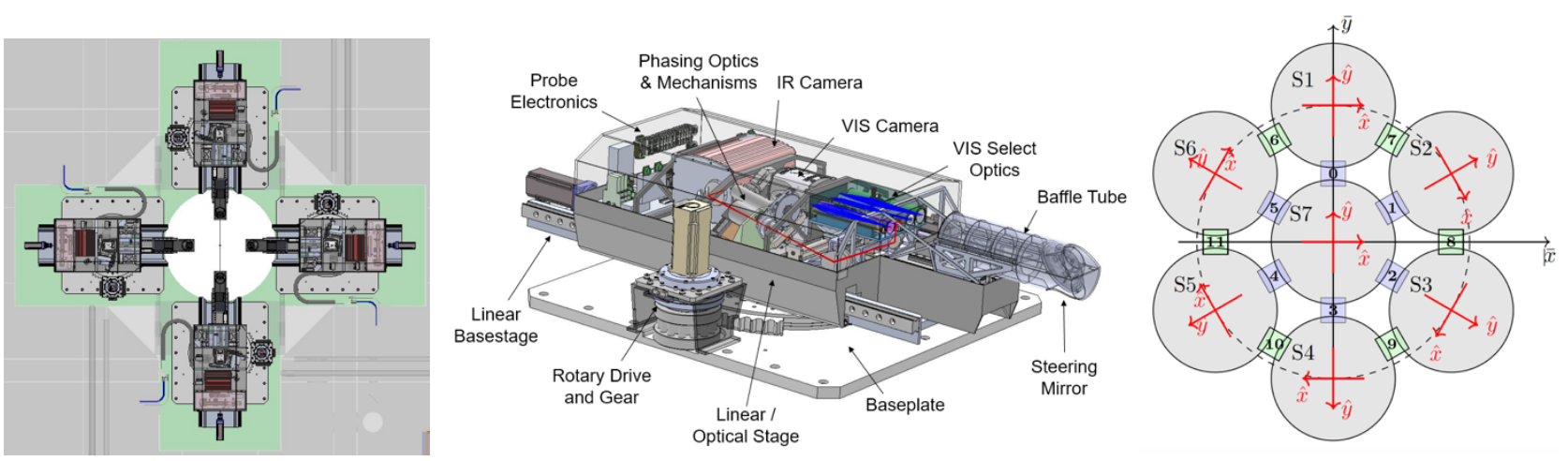

Figure 2. (Left) AGWS system layout with four identical star probes. (Center) Star probe design overview. (Right) Location of the 12 DFS sub-apertures on the GMT pupil. 


\section{PHASING AND ACTIVE OPTICS CONTROL SYNERGY}

We will present in this section the revised control strategy designed to maintain the phased condition across the scientific field of view ( $<90$ " in diameter) when operating the GMT in the diffraction-limited AO modes.

Maintaining the phased condition in the field requires both the relative segment phase piston (SPP) and segment tilt to be controlled, because tilt of an M1 segment compensated by the opposite tilt of its matching M2 segment results in no tilt in the focal plane, but a field-dependent segment phase piston (FDSPP) error. A single off-axis phasing sensor, while accurately measuring the total segment piston at its off-axis position, cannot disentangle a true SPP error from a FDSPP. This is the main reason why the AGWS design features four phasing sensors that can be positioned in the field: to probe the field-dependent component of the segment piston error. Baseline operation is to operate three out of four AGWS phasing sensors simultaneously.

We have previously presented a phasing control algorithm (hereafter referred to as "Algorithm I") that used the phasing sensors in the AGWS to drive the compensation of SPP and FDSPP [5][6]. Algorithm I was prone to estimation errors coming from the presence of M1/M2 aberrations ${ }^{1 *}$ that, when measured by the phasing sensors, were aliased onto SPP and FDSPP. The aliasing of low-order modes occurs because these modes are sampled by the DFS subapertures in just a small portion of the pupil over which the average differential piston may not be zero (see Figure 2).

This aliasing issue prompted us to investigate better algorithms relying on additional wavefront sensors readily available in the AGWS to better cope with both phasing and low-order modes residuals. This new algorithm (hereafter referred to as "Algorithm II") is presented in Section 2.1. The computation of the required reconstructor is described in Section 2.2, and a preliminary validation based on numerical simulations is presented in Section 2.3.

\subsection{Algorithm II. Phasing / Active Optics Control}

Algorithm II is designed to do phasing control while coping better with residual field-dependent aberrations. It can be understood as a generalization of Algorithm I, in which a larger number of field-dependent aberrations (FDA) are estimated, starting from the original one, the FDSPP.

In this control strategy both AGWS visible and phasing channels on each probe are utilized simultaneously. The AGWS visible channel is configured in its "Wavefront Sensing" function, featuring a $48 \times 48$ Shack-Hartmann WFS (referred to as AGWS SH hereafter).

The modes that are sought to be controlled are all M1 segment rigid-body Degrees of Freedom (DoF), i.e. $T_{x y z}, R_{x y z}$, and a subset of bending modes $\left(B_{m}\right)$, except for $T_{z}$ and $R_{z}$ of the central segment (M1S7) which acts as the reference for outer segments. The Phasing/Active Optics control strategy is illustrated in the diagram of Figure 3.

\subsection{AGWS merged reconstructor}

The estimation of all M1 DoF can be done with a single reconstruction matrix $\left(R_{M}\right)$, as indicated in the diagram of Figure 3. We have called $R_{M}$ the AGWS merged reconstructor because it takes as inputs the measurement vectors delivered by both the AGWS DFS and AGWS SH sensors:

$$
\left[\begin{array}{c}
\Delta T_{x y z} \\
\Delta R_{x y z} \\
\Delta B_{m}
\end{array}\right]=R_{M}\left[\begin{array}{l}
\left(\frac{1}{G_{D F S}}\right) \vec{s}_{p} \\
\left(\frac{1}{G_{S H S}}\right) \vec{s}_{h}
\end{array}\right]
$$

The merged AGWS reconstructor is computed as the pseudo-inverse of the merged interaction matrix, denoted as $M_{M}$ :

$$
R_{M}=M_{M}^{\dagger}=\left[\begin{array}{l}
\left(\frac{1}{G_{D F S}}\right) M_{D F S} \\
\left(\frac{1}{G_{S H S}}\right) M_{S H S}
\end{array}\right]^{\dagger}
$$

\footnotetext{
${ }^{1 *}$ We call "M1/M2 aberrations" those aberrations on M1 segments (e.g. Active Optics residuals) that, when the AO loop is closed, are compensated by the corresponding M2 ASM segments. These M1/M2 aberrations are the source of Field-Dependent Aberrations (FDA).
} 


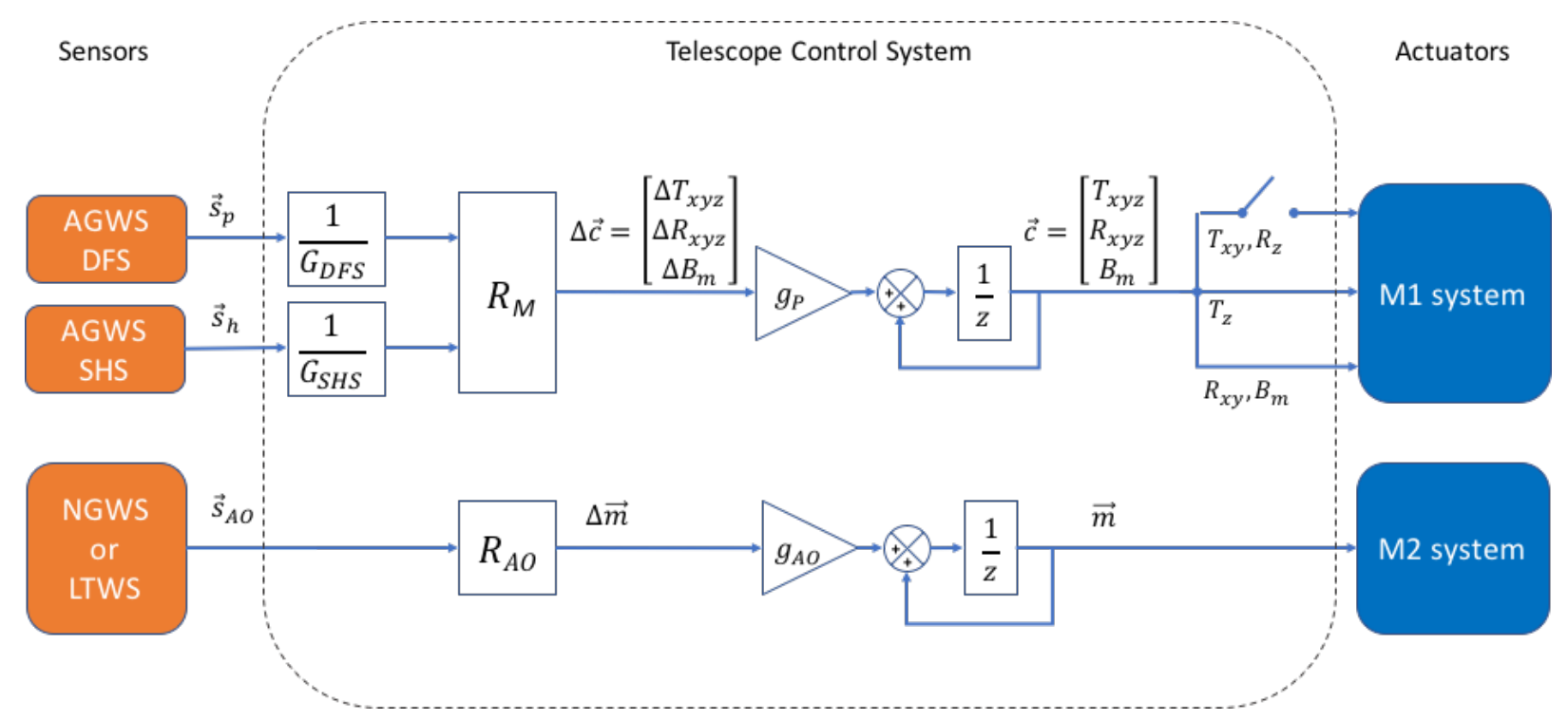

Figure 3. Phasing control strategy using Algorithm II. Two principal control loops are running in parallel: an AO loop driving the shapes of the M2 (ASM) segments, and a Phasing/Active Optics loop driving $\mathrm{T}_{\mathrm{xyz}}, \mathrm{R}_{\mathrm{xyz}}$, and $\mathrm{B}_{\mathrm{m}}$ on M1 segments to compensate for segment piston (SPP) and field-dependent aberrations, comprising field-dependent segment phase piston (FDSPP).

where $M_{D F S}$ is the AGWS DFS interaction matrix, $M_{S H S}$ is the AGWS SH interaction matrix, $G_{D F S}$ and $G_{S H S}$ denote the Frobenius norm of $\mathrm{M}_{\mathrm{DFS}}$ and $\mathrm{M}_{\mathrm{SHS}}$, respectively. These interaction matrices contain the signals generated by M1/M2 fielddependent aberrations on the three (or four) AGWS DFS/SH sensors.

The interaction matrices acquisition procedure is the same as the one used to calibrate the FDSPP in Algorithm I [5]. In brief, the calibration steps are: 1) Apply a positive/negative DoF on an M1 segment; 2) Close the on-axis AO loop driving the ASM to compensate for the introduced M1 DoF; 3) Acquire the AGWS DFS and SH measurements after the previous step converged.

Note that $T_{z}$ can also be in principle calibrated with the LTAO loop closed, but not with the NGAO because the NGWS is sensitive to segment piston and it would remove it completely.

As an example, Figure 4 shows the interaction matrix between three AGWS DFS and M1 bending modes (15 bending modes per segment) for a symmetric asterism of three stars located 8' off-axis. Even if most of these bending modes do not generate a "pure" FDSPP component, they do generate a signal on the DFS subapertures, as we pointed out above. This information, together with the additional information provided by AGWS SH sensors, is sufficient to disentangle true SPP and FDSPP errors from other field-dependent aberrations.

Because the AGWS DFS and SH sensors have different units and scales, we need to normalize each interaction matrix by its Frobenius norm prior to the matrix inversion ( $\S$ Eq. 2). For instance, the Frobenius norm of our example case $M_{D F S}$ is $10^{7}$ times larger than the norm of $\mathrm{M}_{\mathrm{SHS}}$. This normalization needs to be applied to each measurement vector too (§ Eq. 1$)$.

Following with our example case, Figure 5 shows the SVD decomposition of the AGWS merged interaction matrix $\left(M_{M}\right)$. A total of 145 DoFs were calibrated (i.e. $20 \mathrm{M} 1$ segment translations, $20 \mathrm{M} 1$ segment rotations, $15 \times 7=105$ segment bending modes). The last 6 eigenmodes are filtered out from the SVD inversion. It can be shown that each of these filtered modes correspond to combinations of $\left[T_{x}, R_{z}\right]$ on each segment that create, altogether, a global GMT pupil clocking. 

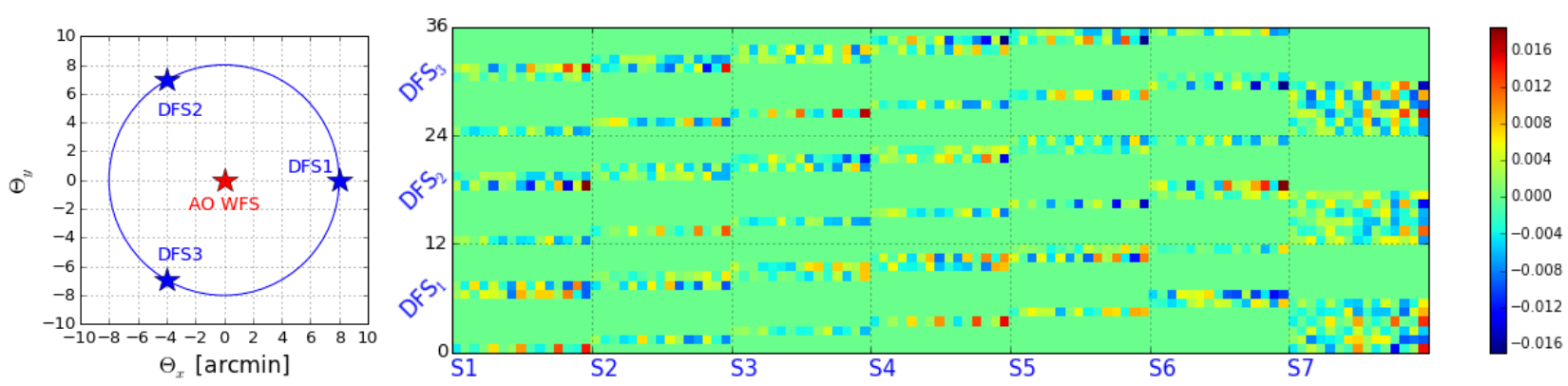

Figure 4. (Left) Wavefront sensing suite comprising an on-axis AO WFS and a set of three Dispersed Fringe Sensors located in a circle of 8' radius; (Right) Interaction Matrix between three AGWS DFS and M1 bending modes (15 modes/segment). The units are in (side lobe) displaced pixels per $10^{-6}$ bending mode coefficient.

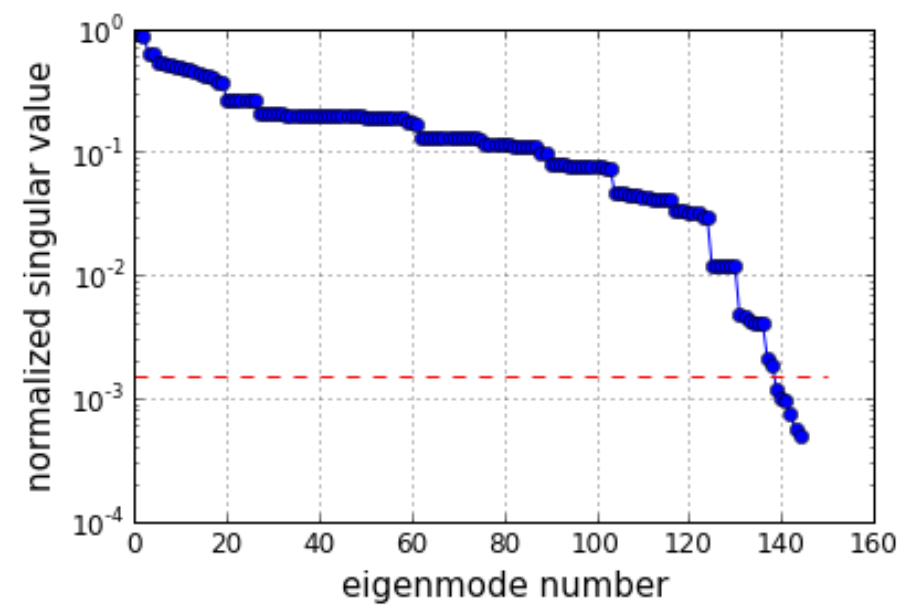

Figure 5. Normalized singular values of the AGWS merged interaction matrix $\left(\mathrm{M}_{\mathrm{M}}\right)$ comprising a total of 145 DoFs (20 M1 segment translations, $20 \mathrm{M} 1$ segment rotations, $15 \times 7=105$ segment bending modes). The red dashed line indicates the threshold chosen to filter out the last 6 eigenmodes.

\subsection{Preliminary validation}

This section presents a preliminary simulation result that validates Algorithm II in the absence of turbulence, dynamic disturbances and measurement noise. Simulations were done with the Dynamic Optical Simulation (DOS) tool [7].

The simulated wavefront sensing configuration is shown on the Left of Figure 4. The two control loops depicted in Figure 3 running in parallel have been simulated: an AO loop driving the ASM, and a Phasing/Active Optics loop running 15 times slower controlling the $145 \mathrm{M} 1 \mathrm{DoF}$ already enumerated. The absolute loop rates are not relevant in this preliminary noiseless simulation.

The AGWS DFS were simulated with end-to-end optical models (see [6] for full description). The AGWS SH were simulated with noiseless geometrical models. NGAO and LTAO end-to-end simulations are in themselves quite complex and time consuming. For the purpose of this study, we decided to simulate a simplified "LTAO-ish" system that provides an equivalent level of on-axis correction. The LTAO-ish simulation uses a single on-axis $60 \times 60$ SH WFS implemented with a geometrical noise-free model, coupled to an on-axis natural guide star. In order to mimic the performance of the real LTAO system, the number of simulated controlled (KL) modes on the ASM was tuned to obtain a SR in K band of $\sim 0.5$ under median seeing conditions.

The goal of this preliminary simulation is to verify that the phasing loop does converge using Algorithm II. The initial (static) disturbance is shown Figure 6(a), featuring an M1 segment scramble of all relevant DoF. Note that the central segment (M1S7) is scrambled too, except for its $T_{z}$ and $R_{z}$, which are not controlled by our AGWS merged reconstructor. 
Also, note that this M1 scramble features segment piston errors of up to $40 \mu \mathrm{m} \mathrm{WF}$, i.e. the limits of the phasing sensors capture range [6].

Figure 6(b, c, d) demonstrates the convergence of the phasing loop with Algorithm II. Note that the WFE on axis converges to zero. Also, because $T_{z}$ and $R_{x y}$ converge to zero, SPP and FDSPP in the whole field are completely removed. There are no aliasing effects coming from other FDA onto these modes.

Figure 7 shows the convergence of all the other rigid-body DoFs controlled in our example simulation. Note that all modes converge to zero, except $T_{x}$ and $R_{z}$, as shown in Figure 7(a) and (f). These combinations of DoF are in fact the eigenmodes filtered out in the SVD inversion ( $§$ Figure 5) which remain uncontrolled. Yet, no image quality degradation is produced by these modes.

We noticed in our first simulations that $T_{x y}$ and $R_{z}$ overshoot when the phasing loop is closed. Therefore, when using Algorithm II, we decided that the commands of these modes will be only applied after a couple of phasing loop iterations. This action is represented in the diagram of Figure 3 as a "switch" on the $T_{x y}, R_{z}$ command.

(a)

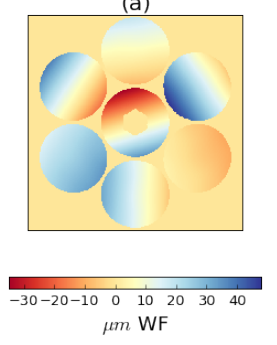

(b)

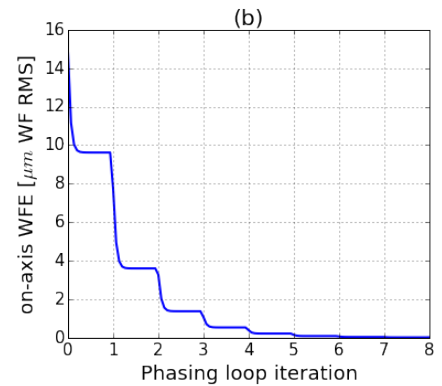

(c)

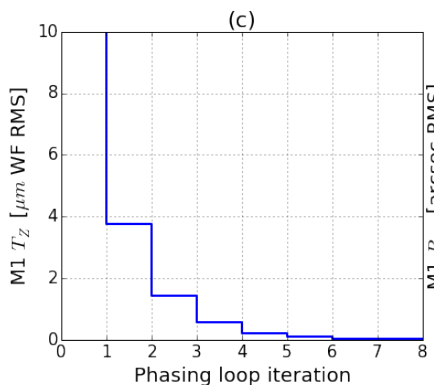

(d)

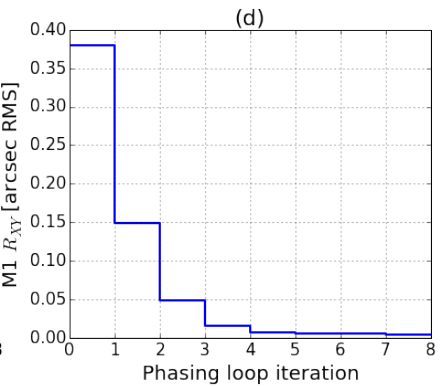

Figure 6. (a) Initial WF with M1 segments scrambled $\left(\sigma_{\mathrm{T}_{\mathrm{z}}}=5 \mu \mathrm{m}\right.$ RMS surface, $\sigma_{\mathrm{R}_{\mathrm{xy}}}=0.38 \operatorname{arcsec} \mathrm{RMS}, \sigma_{\mathrm{T}_{\mathrm{xy}}}=1 \mu \mathrm{m}$ RMS, $\sigma_{\mathrm{R}_{\mathrm{z}}}=0.10$ arcsec RMS, and $\sigma_{\mathrm{B}_{\mathrm{m}}}=5 \mu \mathrm{m}$ RMS coefficients). Phasing loop convergence using Algorithm II: (b) Onaxis WFE vs. phasing loop iteration; (c) M1 $\mathrm{T}_{\mathrm{z}}$ RMS vs. phasing loop iteration; (d) M1 $\mathrm{R}_{\mathrm{xy}}$ RMS vs. phasing loop iteration.
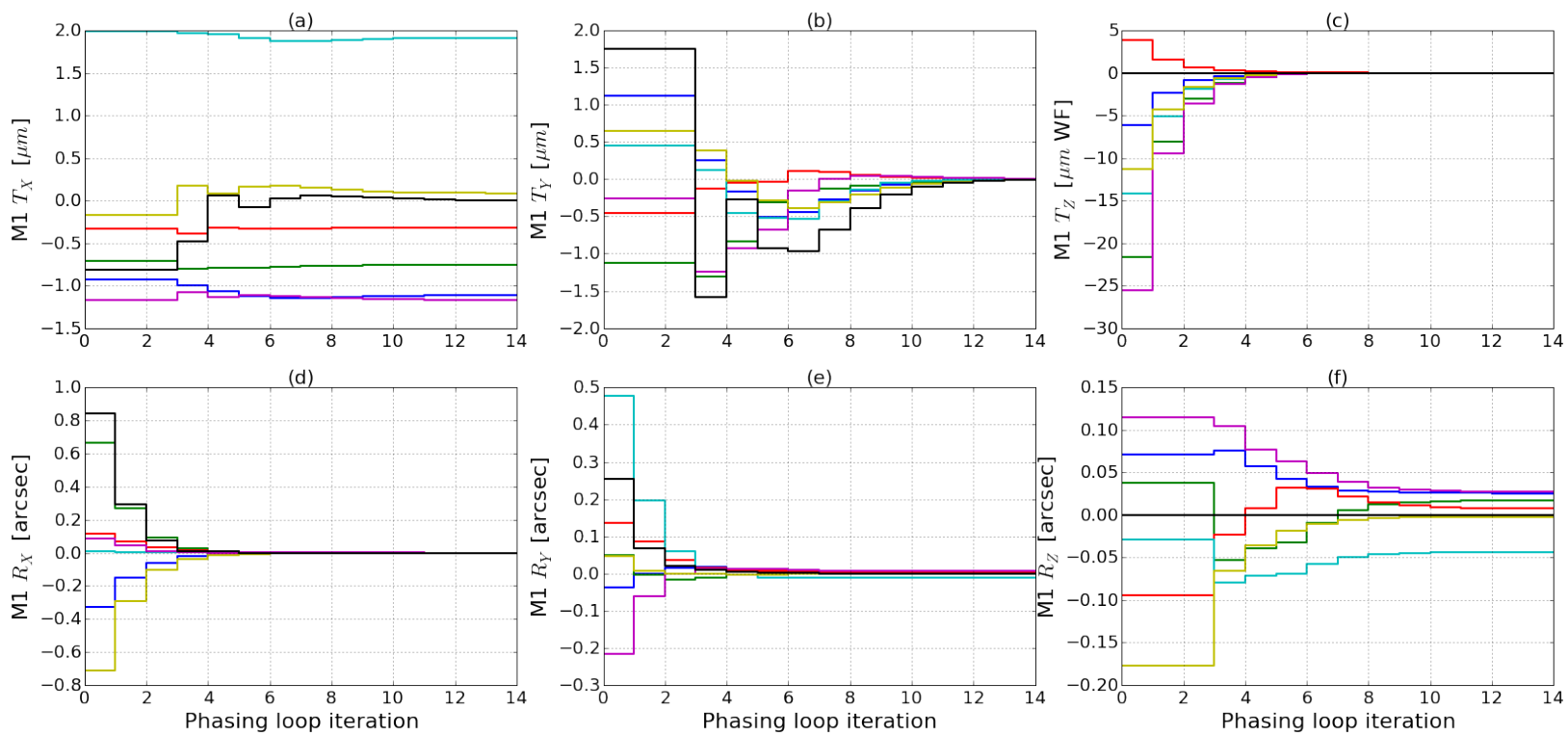

Figure 7. M1 segment DoF convergence example: (a) $T_{x}$ on each segment; (b) $T_{y}$ on each segment; (c) $T_{z}$ on each segment.

(d) $R_{x}$ on each segment; (e) $R_{y}$ on each segment; (f) $R_{z}$ on each segment.

Figure 8 provides additional details on the interaction between the AO and the Phasing / Active Optics loops. The plots show how M1 segment bending modes are being compensated on-axis by the AO loop, generating in that way FDA errors. 
The AO corrections adjust every time Algorithm II removes a fraction of the M1 segment bending modes residuals. In the end, all M1 segment bending modes and M2 segment KL modes converge to zero.

This preliminary simulation shows that Algorithm II, taking advantage of all measurements available from the AGWS SH and DFS sensors, manages to estimate correctly both segment piston and Active Optics residuals, and remove them completely in the absence of noise and time-varying disturbances, while the AO loop is running too. As a next step, we will characterize the performance of this control strategy in more realistic observing conditions.
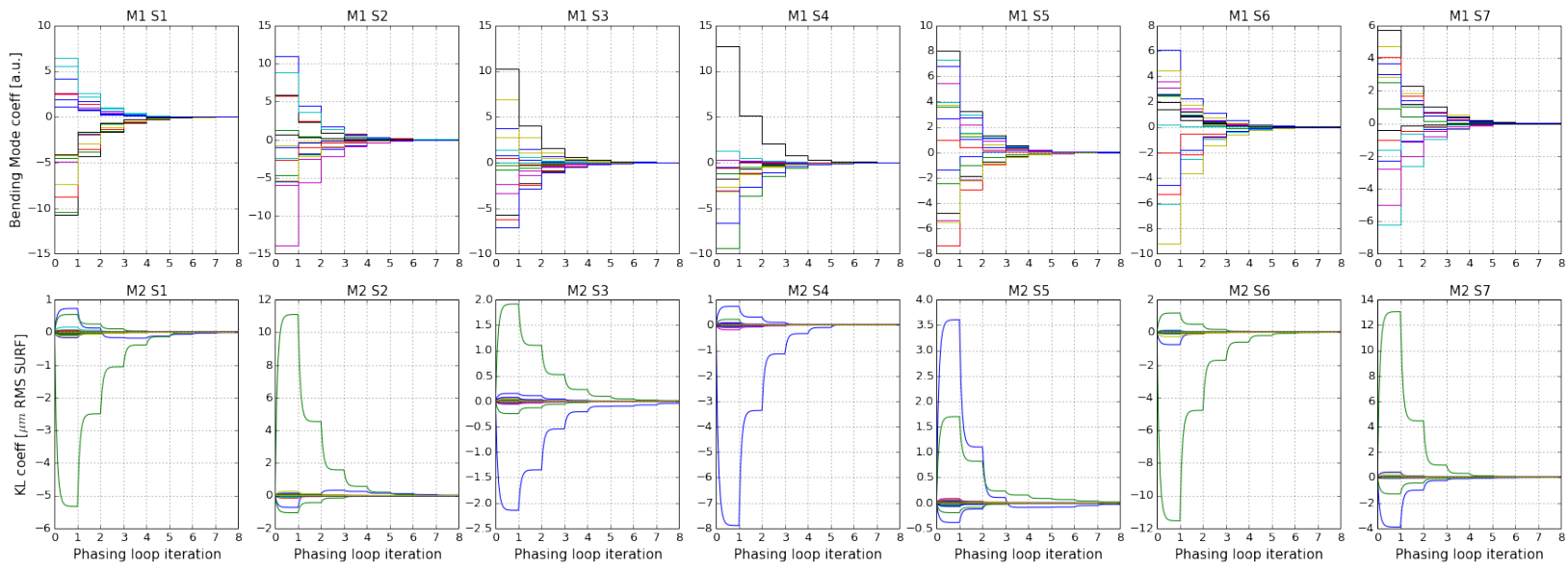

Figure 8. (Top row) Convergence of M1 segment Bending Mode coefficients. (Bottom row) Convergence of M2 KL modes driven by the AO control loop.

\section{WIND BUFFETING REJECTION IN LTAO MODE}

The M1 Edge Sensors (M1ES) and M2 Edge Sensors (M2ES) measure relative piston displacement between segments at high temporal frequency, and are required only in the LTAO mode. Unlike the edge sensors of current telescopes with 1$2 \mathrm{~m}$ hexagonal segments, the M1ES and M2ES will not be used to control segment alignment in closed loop. Instead, they are used exclusively to sense wind buffeting and other high-frequency disturbances of segment optical piston motion, which is then corrected by the ASM through a feed-forward control loop. Figure 9 shows the block diagram representation of the GMT phasing strategy in LTAO mode.

Given the non-zero coefficient of thermal expansion of the GMT M1 segments and changing ambient temperature, it is acknowledged that the M1ES measurements will drift with time. Their reference values will therefore be updated based on the measurements of the AGWS phasing sensors at slow rates $(\sim 0.03 \mathrm{~Hz})$.

Many edge sensor architectures have been evaluated, including metrology bodies which overhang the segments, capacitive sensors between segments, and various types of optical sensors. The selected architecture uses 48 distance-measuring interferometer (DMI) baselines to measure the relative displacement of the segments in each mirror (Figure 10). This DMI arrangement is sensitive to all rigid body degrees of freedom of the outer segments with respect to the on-axis segment, but only the piston measurement is used to control the ASM. It may be possible to use the edge sensor measurements to improve the rejection of segment tip and tilt as well, but this has not yet been evaluated. 


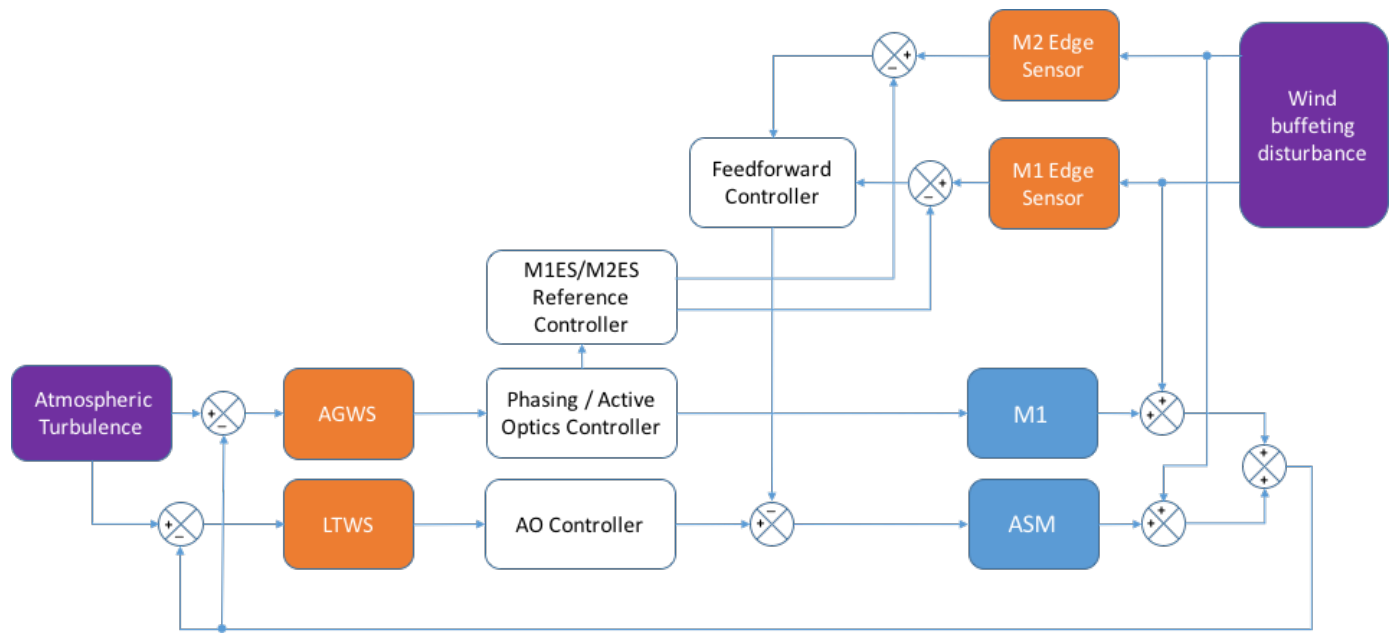

Figure 9. Phasing in LTAO strategy. The AGWS measures low temporal frequency phasing errors. The edge sensors (M1ES and M2ES) measure mid temporal frequency phasing errors mainly due to wind buffeting on M1 and M2 segments, and feedforward the correction to the ASM.

\subsection{Preliminary Validation}

We will report in this section the results of a preliminary analysis to characterize the SPP rejection transfer function (RTF) in LTAO mode. This analysis was limited to the effects of wind buffeting over M1S1 (which is the segment that is the highest when the telescope is tilted, thereby seeing the most wind), and its partial rejection thanks to M1ES measurements fed-forward to the ASM.

We used the M1 off-axis segment dynamic model implemented in Simulink by the GMT Integrated Modelling team. This model can simulate the structural dynamical response of the M1 segment when subjected to internal and external loads (gravity, wind pressures, thermal gradients, etc), and the damping effects of the M1 closed-loop force control system comprising 165 pneumatic actuators and the 6 hard points actuators.

The pressure impinged by the wind over the M1 segment was characterized by a temporal Power Spectral Density (PSD) derived from previous CFD analyses for particular telescope/dome conditions. For our analysis, telescope is pointing at $30^{\circ}$ from zenith, the enclosure vents are $50 \%$ open, and the telescope and enclosure opening are facing directly the incoming wind, which has an outside velocity of $10 \mathrm{~m} / \mathrm{s}$. The pressure PSDs are shown in Figure 11(a). The four PSDs shown in the plot correspond to the four quadrants of the M1 segment. It has been assumed that the pressure over one quadrant is uncorrelated to the pressure over the others.
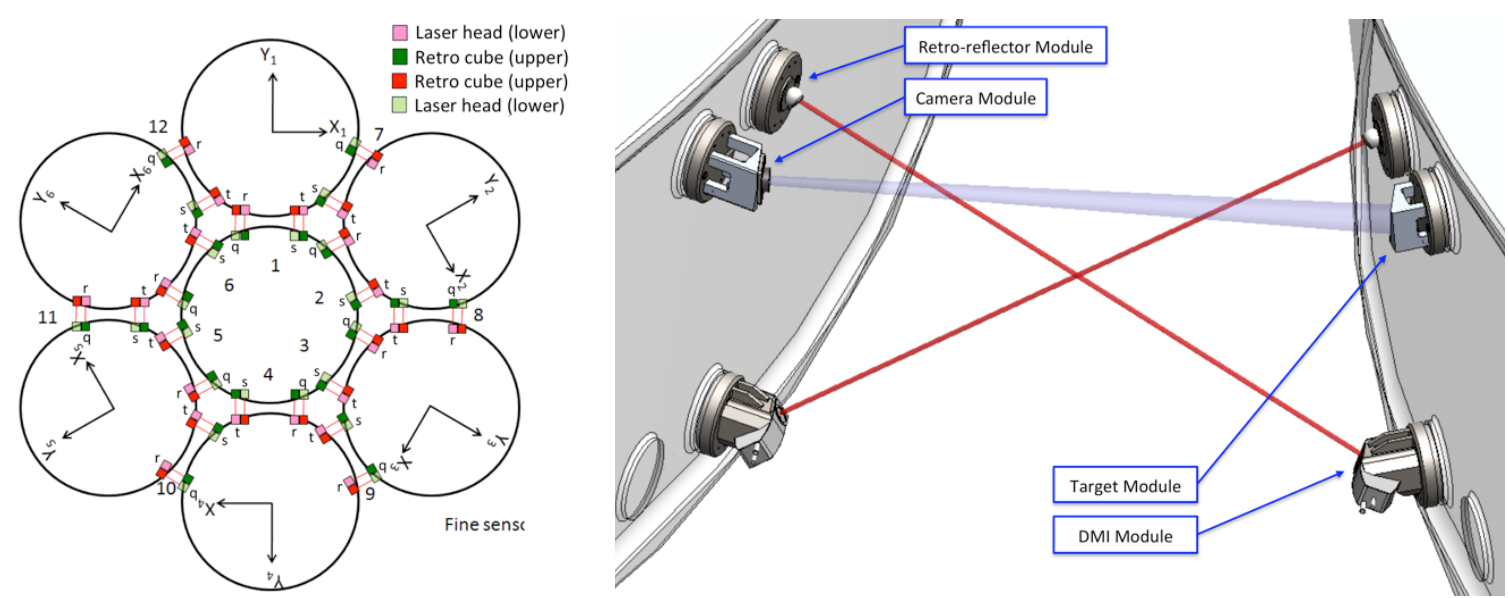

Figure 10: (Left) M1 and M2 edge sensor geometry. (Right) One pair of M1ES distance-measuring interferometers. 


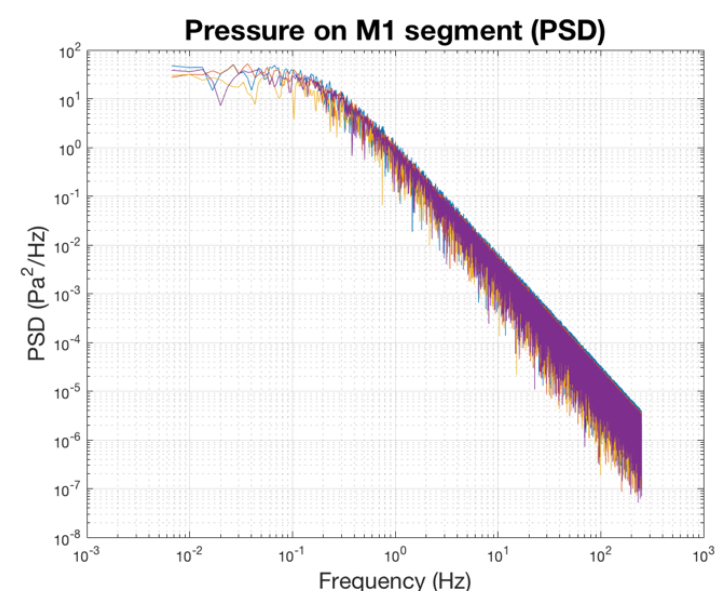

(a)

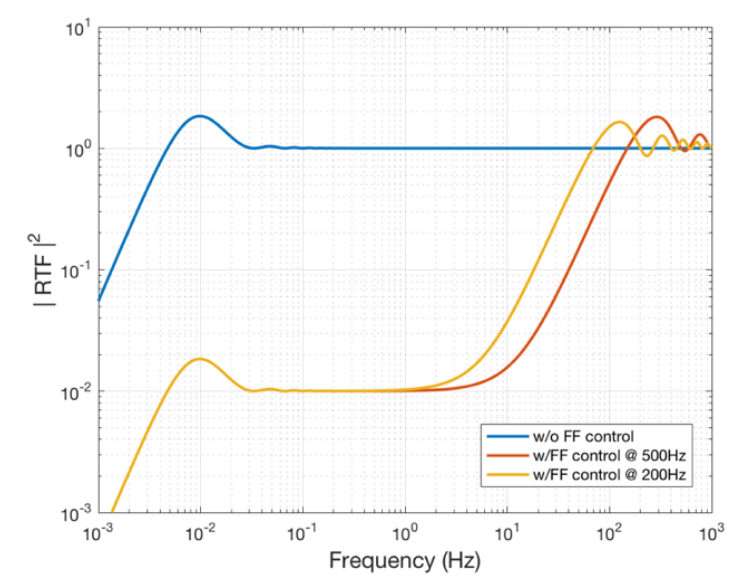

(c)

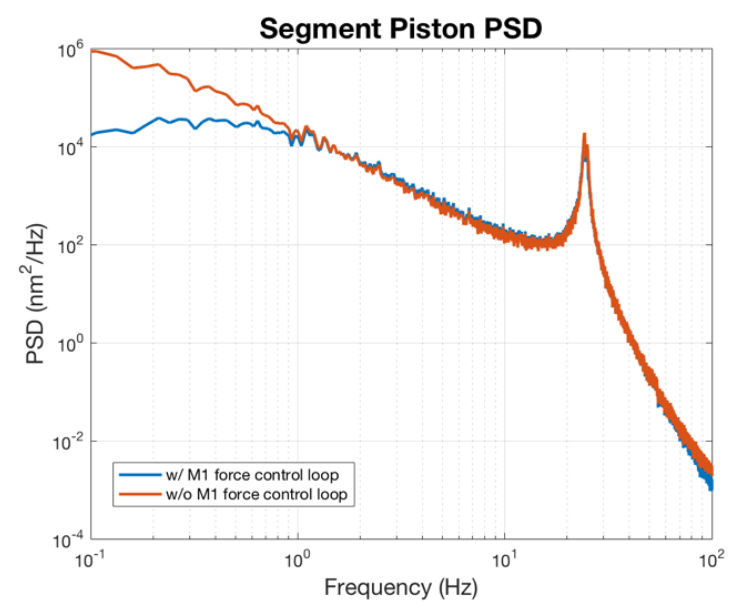

(b)

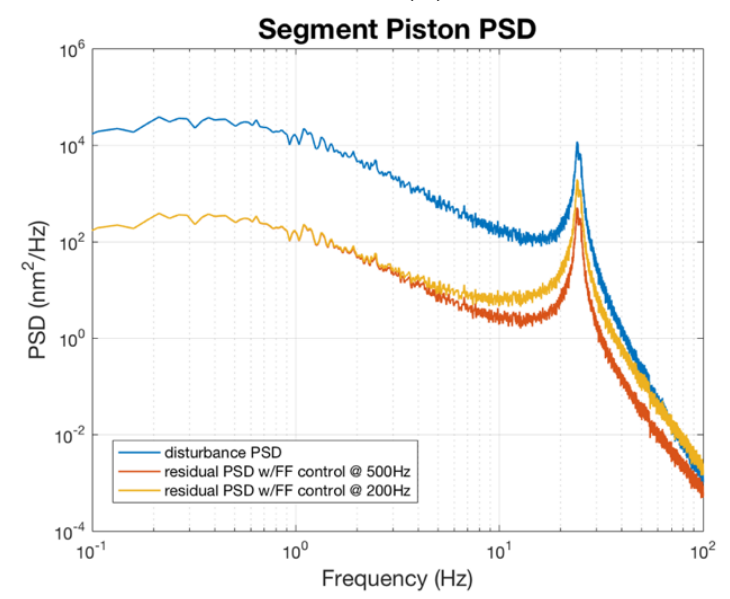

(d)

Figure 11. (a) Pressure power spectral densities (PSDs) over the four quadrants of the M1S1 segment in the described model configuration. (b) SPP PSD of the M1S1 segment due to the wind disturbances, with and without M1 force control loop. (c) Example of SPP rejection transfer functions. (d) Example of Residual SPP PSDs.

We have run a simulation of the M1 dynamic response when subjected to the considered wind pressures in two particular cases: 1) without M1 force control, and 2) with the M1 force control system operating at a frame rate of 500Hz. The resulting SPP PSDs are shown in Figure 11(b). The M1 control system effectively provides an attenuation of the wind disturbances at low $(<1 \mathrm{~Hz})$ temporal frequencies. The SPP error is reduced from $513 \mathrm{~nm}$ WF RMS (without M1 force control) to $242 \mathrm{~nm}$ WF RMS. This SPP error includes a factor $\sqrt{2}$ to take into account that the central segment which acts as the reference will also be jittering in a similar but uncorrelated way. The SPP PSD shows a peak at $\sim 23 \mathrm{~Hz}$, which corresponds to the resonant frequency of the M1 mirror supported by its hardpoints.

We developed in Simulink a Single Input Single Output (SISO) control loop in which the variable of interest is the SPP of M1S1 w.r.t the central segment. The model comprises a slow optical Feed-Back (FB) loop driven by the AGWS, and a fast Feed-Forward (FF) correction driven by the M1ES. Examples of the SPP RTF generated with our Simulink model are shown in Figure 11(c). Without FF control, the Correction BandWidth (CBW), defined as the 0dB cross-over frequency of the RTF, is limited by the large integration time of the AGWS phasing sensor ( $\mathrm{T}=30 \mathrm{~s}$ ) to $\sim 0.005 \mathrm{~Hz}$. FF control significantly increases the $\mathrm{CBW}$ to $\sim 70 \mathrm{~Hz}$ (with update rate at $200 \mathrm{~Hz}$ ) or $\sim 150 \mathrm{~Hz}$ (with update rate of $500 \mathrm{~Hz}$ ) due to the fast albeit open-loop compensation of SPP disturbances. 
The PSD of the residual SPP error, $\mathrm{S}_{\text {res }}(\mathrm{f})$, is simply computed as:

$$
S_{\text {res }}(f)=|\operatorname{RTF}(f)|^{2} S_{\text {in }}(f)
$$

where $S_{\text {in }}(f)$ is the SPP PSD attenuated by the M1 force control system, and RTF(f) is the SPP RTF. The residual SPP PSDs are shown in in Figure 11(d) for the two considered FF control sampling rates. The SPP error is reduced from 242 nm WF RMS to 57 nm WF RMS (@200Hz) or 34 nm WF RMS (@ 500Hz).

In conclusion, FF control based on fast optical edge sensors can improve the phasing performance in the LTAO mode by rapidly suppressing SPP disturbances arising from the wind buffeting over M1 segments. A full validation and estimation of SPP residuals will require including wind buffeting effects on M1, M2 and the full GMT structure. An integrated model of the GMT is currently being developed to address this kind of studies [8]. We will perform full end-to-end numerical simulations to validate segment piston control with the GMT integrated model as a next step.

\section{CONCLUSIONS AND FUTURE WORK}

In this paper we presented a revised Phasing / Active Optics control strategy to be used when the GMT operates in the diffraction-limited adaptive optics modes. The control strategy takes advantage of simultaneous measurements taken by the AGWS infrared phasing sensors and visible Shack-Hartmann sensors to estimate both M1 figure and misalignment errors, comprising segment piston errors. Having all these measurements multiplied by a single "merged" reconstruction matrix avoids aliasing problems identified in our previous works. As a next step, we will characterize the performance of this control strategy under realistic observing conditions.

We have also presented a preliminary analysis of segment piston disturbance rejection enabled by fast optical edge sensors bridging the gaps between M1 and M2 segments (M1ES and M2ES). We have shown that by feeding forward the information to the ASM, segment piston disturbances can be effectively minimized. The analysis presented was limited to a single M1 segment since that was the only dynamic structural model available to us at the time of the analysis. Currently, a full GMT integrated model - comprising the whole telescope structure, M1 and M2 mechanical models, and inner control loops - is being finalized. As a next step, we will implement a full end-to-end simulation of the phasing strategy in LTAO mode using the GMT integrated model subject to wind pressures at different locations of the structure.

\section{REFERENCES}

[1] "Giant Magellan Telescope website", http://www.gmto.org .

[2] Pinna, E., Agapito, G., Quirós-Pacheco, F., et al., "Design and numerical simulations of the GMT Natural Guide star WFS", Proc. SPIE 9148, 91482M (2014).

[3] Conan, R., Bennet, F., Bouchez, A., et al., "The Giant Magellan Telescope Laser Tomography Adaptive Optics System", Proc. SPIE 8447, 84473P (2012).

[4] Van Dam, M. A., McLeod, B. A., Bouchez, A. H., "Dispersed fringe sensor for the Giant Magellan Telescope", Applied Optics Vol. 55, No. 3 (2016).

[5] Quirós-Pacheco, F., Conan, R., McLeod, B. A., et al., "Wavefront control simulations for the Giant Magellan Telescope: Field-dependent segment piston control", AO4ELT4 conference proceedings (2015).

[6] Quirós-Pacheco, F., Conan, R., Bouchez, A., and McLeod, B. A., "Performance of the Giant Magellan Telescope phasing system", Proc. SPIE 9906, 99066D (2016).

[7] Conan, R., Bouchez, A. H., Quirós-Pacheco, F., and McLeod, B. A., "The Giant Magellan Telescope Dynamic Optical Simulation Tool", AO4ELT4 conference proceedings (2015).

[8] Conan, R., Bouchez, A. H., Angeli, G., "Closed-loop GMT integrated modeling", AO4ELT5 conference proceedings (2017). 